\title{
A rare case of solitary fibrous tumour of the bladder
}

\author{
Renan Javier Otta, MD; ${ }^{*}$ Marco Antonio Acosta, MD; ${ }^{*}$ Carlos Gordillo, $M D^{+}$
}

*Department of Urology, Hospital Universitario La Princesa, Madrid, Spain; 'Department of Pathology, Hospital Universitario La Princesa, Madrid, Spain

Cite as: Can Urol Assoc J 2014;8(7-8):e552-3. http://dx.doi.org/10.5489/cuaj.1857 Published online August 11, 2014.

\section{Abstract}

Solitary fibrous tumour of the bladder is a rare mesenchymal neoplasm with a favourable prognosis. Its symptoms are usually secondary to obstructive symptoms rather than hematuria or other findings typical of other bladder neoplasms. We describe a case of solitary fibrous tumour of the bladder and review the literature.

\section{Case report}

A 78-year-old man came to the emergency department because of hematuria and acute urinary retention. A cystoscopy revealed a solitary lesion at the bladder neck. A transurethral resection of the bladder tumour (TURBT) was performed. Pathologic analysis revealed a solitary fibrous tumour of the bladder without atypia. Immunohistochemistry showed positivity to CD34, Bcl-2, CD99 and vimentin and negativity for cytokeratins and S-100 protein. Five months after the first TURBT, the patient returned with recurrence and a repeat TURBT was performed. Pathological exam again revealed a solitary fibrous tumour. The patient is alive at 36 months, after the second TURBT.

\section{Discussion}

Solitary fibrous tumour (SFT) of the bladder is a rare mesenchymal neoplasm. It is classified into 2 forms: pleural and extrapleural. ${ }^{1}$ It should not be confused with mesothelioma which is a tumour derived from the mesothelium. SFT is a mesenchymal tumour with a fibroblastic differentiation. ${ }^{2}$

It was first described by Klemperer and Rabin in 1931, ${ }^{3}$ but the first case in the urinary tract was reported in $1997 .{ }^{4}$ About 15 cases of SFT of the bladder have been described in the literature. ${ }^{5}$
SFT affects both sexes equally, ${ }^{1}$ and most patients are in their fourth to fifth decade. There are two types of SFT. Most are benign and about $10 \%$ to $20 \%$ are malignant. ${ }^{4}$

This type of tumour is usually asymptomatic and presents as a slow-growing well-delineated exophytic mass; therefore, obstructive symptoms are the most common. Furthermore, it can be source of paraneoplastic syndromes, like hypoglycemia secondary to insulin-like growth factor. ${ }^{1,4}$ In our patient, the presentation was typical of any bladder neoplasm.

The pathological analysis renders a proliferation of bland-looking spindly to oval epithelioid cells that form fascicles, between collagen bundles, and a prominent vasculature simulating an hemangiopericytoma (Fig. 1a)., Malignant counterparts are usually hypercellular lesions, cytologic atypia, necrosis and higher frequency of mitoses. ${ }^{1}$ Immunohistochemistry shows positivity to Bcl-2, CD34 (90$95 \%), \mathrm{CD} 99(70 \%)$ and vimentin. It is probable that $\mathrm{BCl}-2$ is more sensitive than CD34 for the diagnosis (Fig. 1b). ${ }^{4}$

Negativity for cytokeratin AE1/AE3, CD31 and S-100 protein is common. ${ }^{1,4}$ In our case, the pathology report yielded positivity to CD34, bcl-2, CD99 and vimentin which strongly suggested a SFT. It was negative for muscle markers, cytokeratin, S-100 protein and anaplastic lymphoma kinase (ALK), which exclude other tumours, such as sarcomas with muscle differentiation, sarcomatoid carcinomas and neurofibromas.

The differential diagnosis should be made with hemangiopericytoma, leiomyoma, nodular fasciitis, inflammatory myofibroblastic tumour, fibromatosis and benign peripheral nerve sheet tumour. Surgery is considered the treatment of choice by local excision or TURBT when symptoms are malignant. ${ }^{1,4}$ Chemotherapy is reserved for metastatic or symptomatic non-resectable malignant tumours. There are no standard chemotherapeutic indications, but the most effective drugs seem to be anthracyclines and ifosfamide. ${ }^{1}$ In our case, chemotherapy was not offered because of its benign histopathology and indolent natural course. 


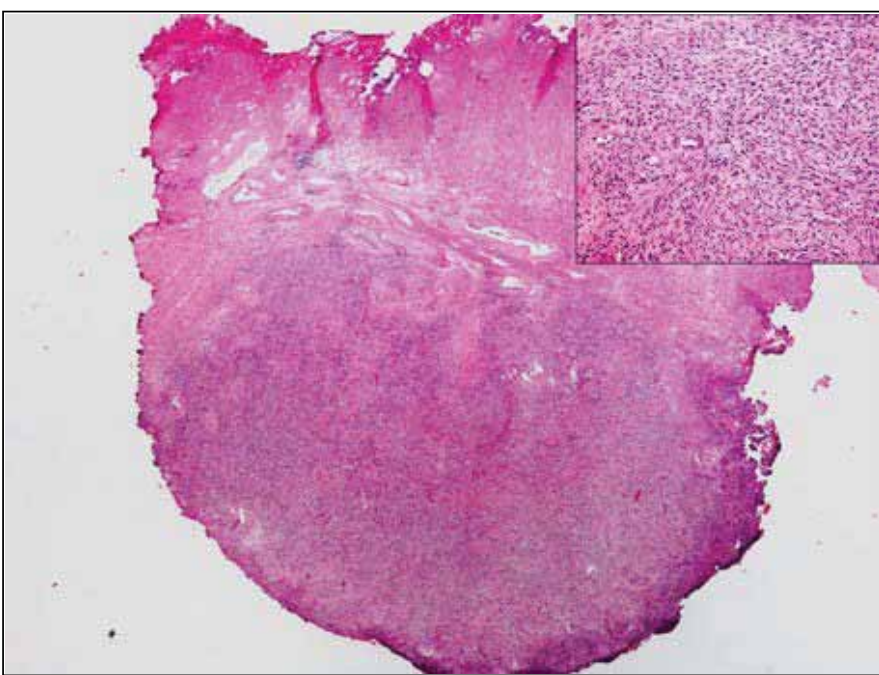

Fig. 1a. Spindle cells arranged in a storiform pattern, zones of hypocellularity and hypercellularity and deposition of intercellular collagen.

The prognosis of this tumour is usually favourable. ${ }^{4}$ The most important prognostic factor is the resectability. ${ }^{1}$ Extrathoracic location, positive surgical margins, tumour size greater than $10 \mathrm{~cm}$ and histopathological appearance were relevant factors for local recurrence and metastasis in a large series of SFT of all locations. ${ }^{6}$ Recurrence occurs in only $10 \%$ to $20 \%$ of cases. $^{3}$

\section{Conclusion}

In our case, the patient recurred 5 months after the initial TURBT. We believe it was because the first resection was sub-total. Since the pathology showed no atypia or malignant component nor was the tumour big $(>10 \mathrm{~cm})$, it was considered unnecessary to search other sites for metastatic spread. Currently our patient is being followed with echography and cystoscopy without evidence of recurrence.

Acknowledgments: The authors would like to thank C. Olivier, MD, PhD; G. Bocardo, MD, PhD; and R. Arellano, MD for their contribution in the preparation of this paper.

Competing interests: Dr. Otta, Dr. Acosta, Dr. Gordillo all declare no competing financial or personal interests.

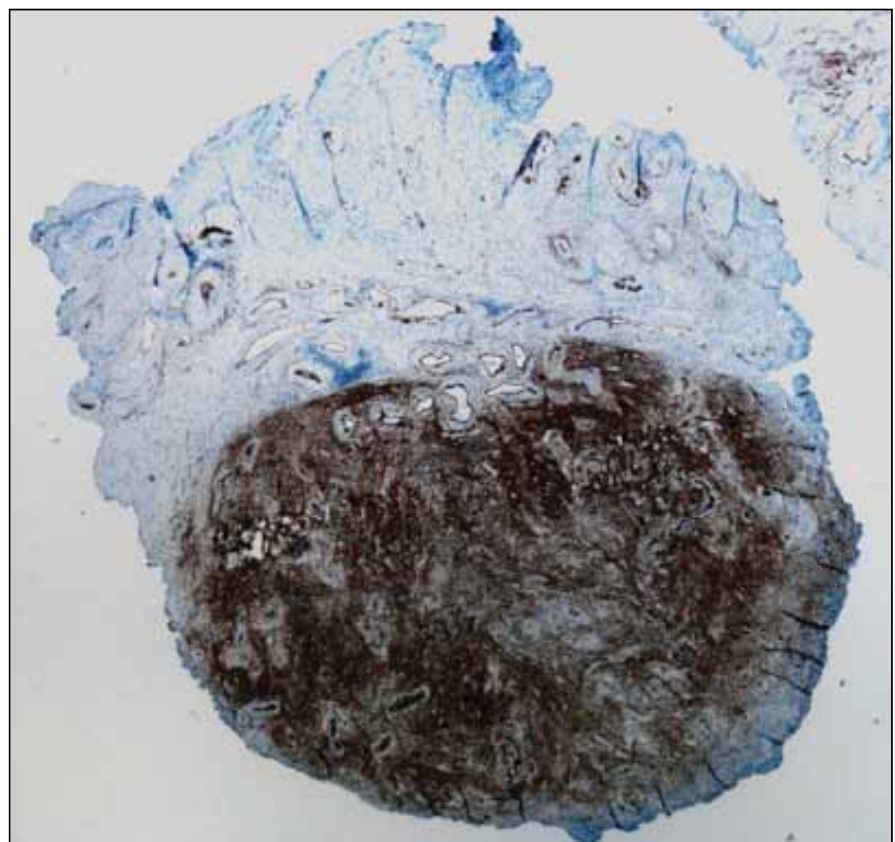

Fig. 1b. Spindle cells proliferation positive for CD 34 marker.

This paper has been peer-reviewed.

\section{References}

1. Bruzzone A, Varaldo M, Ferrarazzo C, et al. Solitary fibrous tumor. Rare Tumors 2010;2:e64.

2. Magi-Galluzzi C, Zhou M, Epstein Jl. Neoplasms of the urinary bladder. In: John R. Goldblum, editor. Genitourinary pathology. Philadelphia, PA: Elsevier; 2007:208-9.

3. Klemperer P, Rabin CB. Primary neoplasms of the pleura. A report of five cases. Arch Pathol 1931;11:385412.

4. López Martín L, Calahorra Fernández FJ. Solitary fibrous tumor of the bladder [in Spanish]. Actas Urol 2010;34:206-17.

5. Seike K, Kameyama K, Kato T, et al. Solitary fibrous tumor. Hinyokika Kiyo 2012;58:105-8.

6. Gold JS, Antonescu CR, Hajdu C, et al. Clinicopathologic correlates of solitary fibrous tumor. Cancer 2002:94:1057-68

Correspondence: Dr. Renan Javier Otta, Department of Urology, Hospital Universitario La Princesa, Madrid, Spain; renan44@gmail.com 